\title{
Texture Analysis of MR Images of Minocycline Treated MS Patients
}

\author{
Yunyan Zhang ${ }^{1,4}$, Hongmei Zhu ${ }^{4}$, Ricardo Ferrari ${ }^{4}$, Xingchang $\mathrm{Wei}^{4}$, \\ Michael Eliasziw ${ }^{3}$, Luanne M. Metz ${ }^{3}$, and J. Ross Mitchell ${ }^{2,3,4}$ \\ ${ }^{1}$ Department of Medical Science, University of Calgary, 2500 University Drive, Calgary, \\ Alberta, Canada T2N 1N4 \\ yunyzhan@ucalgary. Ca \\ ${ }^{2}$ Department of Radiology, University of Calgary, Foothills Medical Centre, Calgary, Alberta, \\ Canada T2N 2T9 \\ rmitch@ucalgary.ca \\ ${ }^{3}$ Department of Clinical Neurosciences, University of Calgary, Foothills Medical Centre, \\ Calgary, Alberta, Canada T2N 2T9 \\ \{eliasziw, lmetz\} @ucalgary.ca \\ ${ }^{4}$ Seaman Family MR Research Centre, Foothills Medical Centre, Calgary, Alberta T2N 2T9 \\ \{hzhu, ferrari, weix\}@ucalgary.ca
}

\begin{abstract}
Texture analysis was applied to 3T brain MR images of 5 relapsing remitting MS patients before and after minocycline treatment. Texture features were used to quantify pathological changes that may be undetectable by conventional MR techniques. Active, inactive lesions and NAWM were chosen from MR images of MS patients one month before and six months after treatment. Texture features changed in all of the selected ROIs. Texture change in active lesions was greater than that in inactive lesions and NAWM. There was close relationship between texture changes in active lesions and NAWM. This preliminary work suggests that it may be possible to predict therapeutic response of MS patients using MRI texture analysis.
\end{abstract}

\section{Introduction}

Multiple sclerosis (MS) is a chronic inflammatory demyelinating disease of the central nervous system afflicting nearly 1 million people worldwide. The hallmarks of multiple sclerosis include multifocal perivascular mononuclear cell infiltrates in the central nervous system, oligodendrocyte loss and demylination, and axonal loss [1]. Recent studies[2,3] revealed that the pathogenesis of MS might be related to the recruitment of autoreactive $\mathrm{T}$ lymphocytes to the central nervous system (CNS) that mediate injury. Therefore, preventing inflammatory cells from infiltrating the CNS may be a reasonable therapeutic strategy for MS [1].

Minocycline is a second-generation of tetracycline. In addition to its known antimicrobial actions, minocycline can inhibit matrix metalloproteinases (MMPs) produced by inflammatory cells, microglial activation, as well as inhibit the activation and proliferation of microglia which are key factors in the pathogenesis of MS and 
EAE (an animal model of MS).[4, 5, 6] We are currently conducting a clinical trial using minocycline for the treatment of patients with MS.

Magnetic resonance imaging (MRI) has become an increasingly important tool for monitoring the natural progression of multiple sclerosis and for evaluating therapeutic response. The most widely used MRI measures are those reflecting disease activity (new or enlarged T2 lesion count, enhancing and new enhancing lesion count, and enhancing lesion volume measurement) and accumulated disease burden (T2 lesion load assessment). Magnetization transfer (MT) MRI holds substantial promise to provide good surrogate measures for MS evolution, especially in phase II and phase III trials for relapsing remitting MS (RRMS) and secondary progressive MS (SPMS).[7] The prominent abnormalities and response to clinical trials can be imaged using the above MR techniques.[8] However, there is an increasing requirement for more rapid and accurate quantification of MRI abnormalities, and the need to improve correlations with clinical course. The use of objective computer-based image analysis to automatically or semi-automatically [9] extract diagnostically significant image features, which can be used to distinguish normal from pathological tissue and to further characterize the state of the pathological tissue, is of critical importance. Texture analysis is one of the techniques that may be utilized to detect subtle changes in tissue and predict the early response of patients to clinical trials.

Texture analysis was initially used to analyze satellite photos. Medical applications of computerized texture analysis dates back to the early 1970s. Intuitively, texture refers to the distribution of brightness and darkness within an image and to concepts such as coarseness, linearity, and regularity. Texture analysis is the term used for methods developed to quantify image texture. Texture analysis methods evaluate the spatial location and signal intensity characteristics of the fundamental structural elements (pixels) of digital images.[10] Texture analysis methods can be categorized into structural, statistical, model-based and transform-based methods.[11] Statistical approaches represent texture indirectly by the non-deterministic properties that govern the distributions and relationships between grey levels in an image. Methods based on second-order statistics had been shown to achieve higher discrimination rates than the power spectrum (transform-based) and structural methods.[12]

Lerske et al [13] performed texture analysis to characterize human brain tissues and segmented white matter, grey matter, cerebral spinal fluid and tumor based on the texture features extracted from tissue co-occurrence matrices.[14] Mathias et al [10] applied similar texture analysis to MR images of spinal cord in MS patients. They showed that there were apparent texture differences between MS lesions and normal appearing white matter (NAWM), and that it was possible to use texture analysis to predict long-term disability. Vince et al [15] compared different texture analysis techniques in characterization of coronary plaques on intravascular ultrasound images, and indicated that Haralick's method [14] yielded the most accurate results. But the follow up of MS patients after clinical trial using texture analysis has not been performed. Based on the relationship between texture feature and structure, one can expect that the changes in texture features may reflect the changes in tissue structure, and the changes in texture may happen earlier than large-scale changes shown by conventional MR images. The goal of this study is to compare the texture changes among different lesions and NAWM before and after minocycline treatment in RRMS patients using Haralick's [14] texture analysis technique retrospectively. 


\section{Patients and Methods}

\subsection{Patients and MR Images}

Five active definite RRMS patients (four females, one male) who had both active and inactive lesions were chosen. Patients aged from 30 to 45 years old with EDSS 0 to 5 . T2-weighted MR images acquired one month before and six months after minocycline treatment were selected.
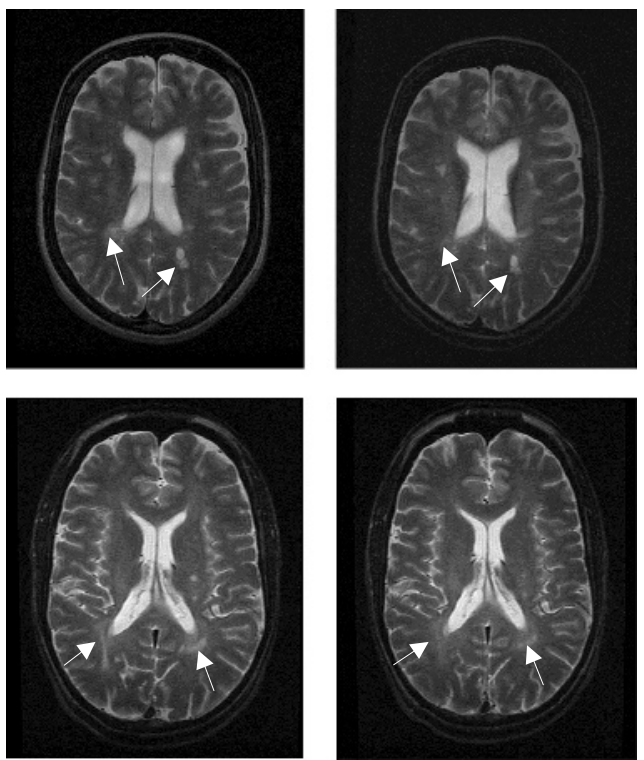

Fig. 1. 3T MR images from two patients in this study. Each row is for one patient. Images in the left column show the lesions (arrows) before treatment, while images in the right column show the same lesions after six months of minocycline treatment. The patient on the top row had both active (left arrow) and inactive (right arrow) lesions, the other patient had only inactive lesions (arrows)

The MR images were acquired from our 3T MR scanner (Signa; GE Medical Systems, Waukesha, WI) using a dual spin echo sequence with TR=2716ms, TE $=20 \mathrm{~ms}$ (proton-density weighted) $/ 80 \mathrm{~ms}$ (T2-weighted), flip angle $=90^{\circ}$, matrix size $=512 * 512$, field of view $=24 \mathrm{~cm}$, spatial resolution $=1.25 \mathrm{~mm}$ and slice thickness $=3 \mathrm{~mm}$ without gap. Examples of 3T MR images with active and inactive lesions in patients with MS are shown in Fig. 1.

All the selected MR images were non-uniformity corrected using the $\mathrm{N}^{3}$ algorithm [16] before image processing. Regions of interests (ROIs) were chosen based on the lesion size and lesion consistency between the two time points for each patient. Only lesions with size of at least 100 pixels were selected. The ROIs for active and inactive lesions and NAWM were placed by two radiologists on the same slice at the same time point. There were five ROIs for active lesions, inactive lesions and NAWM respectively in all five patients both before and after treatment. 
The pixel intensity grey levels in ROIs were normalized to 0 50 to avoid sparse matrices and to reduce computation time. Normalization was done by scaling all gray levels in each ROI between the minimum and maximum onto the range [0...50]. The co-occurrence matrix was then calculated for each ROI using Haralick's method [14].

\subsection{Statistical Texture Features}

Two first order statistical features, mean grey level (MGL) and variance of grey level (VGL) were calculated for each ROI as defined by the following equations, where $\mathrm{G}$ is the number of gray levels in ROI.

$$
\begin{array}{ll}
\text { Mean gray value: } & \mu={ }_{i=0}^{G-1} i p(i) \\
\text { Variance of gray value: } & \sigma^{2}={ }_{i=0}^{G-1}(i-u)^{2} p(i)
\end{array}
$$

Second-order statistics were based on the co-occurrence matrix $h_{d \Theta}(i, j)$. After $h_{d \Theta}(i, j)$ was constructed, it was normalized by dividing each entry by the total number of neighboring pixels in the matrix. This normalized matrix $p_{d \Theta}(i, j)$ then became the estimation of the joint probability of two pixels, a distance $d(d=1$ was chosen in this study) apart along a given direction $\Theta$ having particular (co-occurring) values $\mathrm{i}$ and $\mathrm{j}$. Four second-order texture features: angular second moment (ASM); contrast (CON); correlation (COR); and, entropy (ENT), were calculated from the co-occurrence matrix in each direction according to the following equations. Briefly, the ASM is a measure of homogeneity; the CON is a measure of local contrast; the COR is a measure of gray-tone linear-dependencies and the ENT is a measure of the complexity of the image.

ASM:

$$
\underset{i=0 \quad j=0}{G-1 G-1}[p(i, j)]^{2}
$$

$$
\underset{i=0}{G=0}(i-j)^{2} p(i, j)
$$

$\mathrm{CON}$ :

COR:

$$
\left.\underset{i=0 j=0}{(i j p}(i, j)-\mu_{x} \mu_{y}\right) \div \sigma_{x} \sigma_{y}
$$


ENT:

$$
G-1 G-1
$$

$$
-{ }_{i=0}{ }_{j=0} p(i, j) \log _{2}[p(i, j)]
$$

Where $\mu_{x}, \mu_{y}, \sigma_{x,} \sigma_{x}$ denote the mean and standard deviation of the row and column sums of the matrix respectively.

Each second-order statistical feature was determined in four directions $(0,45,90$ and 135 degrees). The features along each direction were then averaged to create a single overall measure for each feature. This step was repeated for every ROI.

\subsection{Statistics}

A student-t test was used to evaluate both the differences between texture features before and after minocycline treatment, and the magnitude of changes in texture features between active and inactive lesions.



Fig. 2. The magnitude and direction of overall mean changes in texture features for active and inactive lesions and NAWM before and after minocycline treatment. Each group of columns represents one feature. Texture features changes were larger in active lesions than in inactive lesions. In fact, only the variance of gray level appeared to drop in active lesions. There was also change in texture of NAWM after treatment

\section{Results}

Fig. 2 illustrates the mean texture change in active, inactive lesions and NAWM. The increase or decrease of different features was different in different tissues. Texture changes after treatment were greater in active lesions than in inactive lesions. How- 
ever, these changes were not quite significant $(\mathrm{p}=0.07)$. Texture change in NAWM was greater than that in inactive lesions, but less than that in active lesions. Table 1 shows the feature values in the texture of active lesions before and after minocycline treatment in all 5 patients.

The ASM, CON, COR and MGL in active lesions and NAWM changed in the same direction and at a correlated magnitude.

Table 1. The texture features in active lesions before and after treatment in all of the 5 patients. -: before treatment; + : after treatment

\begin{tabular}{llcllll}
\hline & & Pat. 1 & Pat. 2 & Pat. 3 & Pat. 4 & Pat. 5 \\
\hline \multirow{2}{*}{ ASM } & + & 8.55 & 9.18 & 10.6 & 8.85 & 9.71 \\
& - & 11.6 & 8.95 & 10.7 & 9.16 & 9.64 \\
CON & + & 7.4 & 4.7 & 3.2 & 3.0 & 3.1 \\
& - & 5.9 & 7.7 & 9.1 & 3.7 & 2.1 \\
COR & + & 7.1 & 3.9 & 3.1 & 3.7 & 2.7 \\
& - & 3.5 & 7.8 & 4.9 & 4.3 & 2.5 \\
ENT & + & 0.294 & 0.294 & 0.290 & 0.294 & 0.291 \\
& - & 0.287 & 0.293 & 0.289 & 0.293 & 0.291 \\
MGL & + & 28 & 22 & 17 & 21 & 18 \\
& - & 26 & 28 & 31 & 21 & 19 \\
VGL & + & 10.1 & 31.6 & 59.1 & 10.8 & 129.8 \\
& - & 97.9 & 40.1 & 5.2 & 29.8 & 11.0 \\
\hline
\end{tabular}

\section{Discussion}

\subsection{Texture Change in Active Lesions}

The 3T MR images of the five active definite RRMS patients were analyzed retrospectively. All the ROIs in MS lesions and NAWM were marked by two radiologists from T2-weighted MR images before and after minocycline treatment. Minocycline is a commonly used tetracycline with an excellent safety record. As Brundula [1] pointed out, minocycline can inhibit the matrix metalloproteinases (MMPs) enzymatic activity, decreases MMP levels in $\mathrm{T}$ cells, attenuate $\mathrm{T}$ cell migration in vitro, and reduce clinical severity, neuroinflammation and demyelination in EAE. MMPs can help leukocytes to break down the blood brain barrier to enter the CNS. Thus minocycline is hypothesized to target active lesions more efficiently by inhibiting MMPs. We have unpublished results that suggest that GD-enhancing lesions in T1-weighted postcontrast MR images disappear 2-3 months after minocycline treatment. This may suggest a mechanism for the relatively large texture changes in active lesions. 


\subsection{The Diversity of Texture Change}

The increased homogeneity and decreased complexity in texture after minocycline treatment in active lesions and NAWM may be the result of their therapeutic response to minocycline. But the complex changes, such as the increase in contrast, correlation and mean gray level in active, inactive lesions and NAWM, the decrease of variance of gray level in active lesions and increase in inactive lesions and NAWM, may also result from heterogeneity in the pathogenesis in MS. The same lesion pattern in different patients at different stages may also have different structure. However, there was wide variability in this study because of the small sample size (5 ROIs in each category within the 5 patients).

\subsection{Texture Change in NAWM}

There was close relationship between texture changes in active lesions and in NAWM in this study. The change in homogeneity, contrast and mean gray level in NAWM had the same direction as that in active lesions. Also, the magnitude of change was proportional to the change in active lesions. This suggests that NAWM and active lesions might have similar signal abnormality, and a similar response to minocycline treatment. Werring et al [17] showed that there were diffuse abnormalities in the NAWM of MS patients. In an attempt to confirm these effects, it will be necessary to combine more texture features extracted from co-occurrence matrices, analyze results from a larger group of patients, and monitor the patients' therapeutic response over a longer period of time.

\section{Summary}

This study showed that there were differences in the magnitude and direction of changes in texture between active lesions and inactive lesions after minocycline treatment. There was also a close relationship in the texture change of active lesions and NAWM, which indicate that there may be some similar therapeutic response between them. This preliminary work showed that it might be possible to predict the therapeutic response in patients with MS using MRI texture analysis.

\section{References}

1. Brundula, V., Rewcastle, N. B., Metz, M. L., Bernard, C. C., and Yong. V. W.: Targeting leukocyte MMPs and transmigration minocycline as a potential therapy for multiple sclero sis. Brain. 125 (2002) 1297-1308

2. Hohlfeld, R.: Biotechnological agents for the immunotherapy of multiple sclerosis. Principles, problems and perspectives. Brain. 120 (1997) 865-916

3. Noseworthy, J. H,, Lucchinetti, C., Rodriguez, M., Weinshenker, B. G.: Multople sclerosis. New Engl J Med. 343 (2000) 938-952 
4. Yrjanheikki, J., Keinanen, R., Pellikka, M., et al.: Tetracyclines inhinit microglial activation and are neuroprotective in global brain ischemia. Proc Natl Acad Sci USA. 95 (1998) $15769-15774$

5. Tikka, T., Fiebich, B. L., Goldsteins, G., et al.: Minocycline, a tetracycline derivative, is neruoprotective against excitotoxity by inhibiting activation and proliferation of microglia. J Neurosci. 21 (2001) 2580-2588

6. Popovic, N., Schubart, A., Goez, B. D., Zhang, S. C., Linington, C., and Duncan, I. D.: Inhibition of autoimmune encephalomyelitis by a tetracycline. Ann Neurol. 51 (2002) 215223

7. Filippi, M., Tortorella, C., Rovaris, M.: Magnetic resonance imaging of multiple sclerosis. J Neuroimaging. 12 (2002) 289-301

8. Miller, D. H., Grossman, R. I., Reingold, S. C., and McFarlan, F.: The role of magnetic resonance techniques in understanding and managing multiple sclerosis. Brain. 121 (1998) $3-24$

9. Mitchell, J. R., Karlik, S. J., Lee, D. H., Fenster, A.: Computer-assisted identification and quantification of multiple sclerosis lesions in MR imaging volumes in the brain. The Journal of Magnetic Resonance Imaging. 4 (1994) 197-208

10. Mathias, J. M., Tofts, P. S., and Losseff, N. A.: Texture analysis of spinal cord pathology in multiple sclerosis. Magnetic Resonance in Medicine. 42 (1999) 929-935

11. Materka, A., Strzelecki, M.: Texture analysis methods-A review. Technical university of lodz, institute of electronics, COST B11 report, Brussels (1998)

12. Weszka, J., Deya, C., and Rosenfeld, A.: A comparative study of texture measures for terrain classification. IEEE Trans. System, Man and Cybernetics. 6 (1976) 269-285

13. Lerski, R. A., Straughan, K., Schad, L. R., Boyce, d., Bluml, S., and Zuna, I.: MR image texture analysis - an approach to tissue characterization. Magnetic resonance imaging. 11 (1993) 873-887

14. Haralick, R. M., Shanmugam, K., and Dinstein, j.: Textural features for image classification. IEEE Trans Syst Man Cybern. 6 (1973) 610-621

15. Vince, D. G., Dixon, K. J., Cothren, R. M., Cornhill, J. F.: Comparison of texture analysis methods for the characterization of coronary plaques in intravascular ultrasound images. Computerized medical imaging and graphics. 24 (2000) 221-229

16. Arnold, B. J., Liow, J., Schaper, A. K., Stern, J. J., Sled, G. J., Shattuck, W. D., Worth, J. A., Cohen, S. M., Leahy, M. R.: Qualitative and quantitative evaluation of six algorithms for correcting intensity nonuniformity effects. NeuroImage. 13 (2001) 931-943

17. Werring, D. J., Clark, C. A., Barker, G. J., Thompson, A. J., Miller, D. H.: Diffusion tensor imaging of lesions and normal-appearing white matter in multiple sclerosis. Neurology. 52 (1999) 1626-1632 